\title{
Uso de fator protrombínico no tratamento de hematoma subdural espontâneo: relato de caso
}

\section{Prothrombin Factor Use in the Treatment of Spontaneous Subdural Haematoma: Case Report}

\author{
Marco de Agassiz Almeida Vasques ${ }^{1}$ \\ ${ }^{1}$ Neurocirurgião, Centro Médico Total Care, Hospital das Forças \\ Armadas, Brasília, DF, Brasil \\ Arq Bras Neurocir 2016;35:67-69.
}

\begin{abstract}
Address for correspondence Marco de A.A. Vasques, MD, Hospital das Forças Armadas, Serviço de Neurocirurgia, SHS Quadra 04 Bloco G, Brasília, DF, Brasil CEP: 70314-000 (e-mail: mvasques.ncr@ig.com.br).
\end{abstract}

\section{Resumo \\ Palavras-chave \\ - varfarina \\ - hemorragia subdural \\ - protrombina}

A warfarina é amplamente utilizada na condução de pacientes com patologias cardiovasculares, apesar do risco de sangramentos potencialmente graves. Quando tais sangramentos ocorrem, é necessária rápida reversão da anticoagulação. No presente relato descreve-se um paciente que desenvolveu hematoma subdural espontâneo associado ao uso de warfarina, necessitando intervenção cirúrgica emergencial. É dada ênfase aos mecanismos de produção deste fenômeno e à sua condução clínica.

Warfarin is widely used on the treatment of cardiovascular pathology, although there are risks of potentially lethal bleeding. When such bleeding occurs, it is imperative a rapid reversal of anticoagulation. A case of a spontaneous subdural hematoma during the use of warfarin and requiring emergent surgical treatment is reported. Emphasis is given to the mechanisms of this phenomenon and its clinical conduction.

\section{Introdução}

A warfarina é um anticoagulante amplamente utilizado em patologias cardiovasculares. ${ }^{1}$ Sua utilização traz o risco de sangramentos potencialmente graves. ${ }^{1,2}$ No presente relato descreve-se um paciente que desenvolveu hematoma subdural espontâneo na vigência do uso desta medicação, necessitando intervenção cirúrgica emergencial com utilização de fator protrombínico para reversão da anticoagulação.

\section{Materiais e Métodos}

Paciente de 69 anos, sexo masculino, militar, com antecedente de infarto agudo do miocárdio ocorrido no ano de 2004, tratado com colocação de dois stents coronarianos e uso de warfarina (Marevan) desde então. Foi admitido no serviço em 17 de março de 2013 com o relato de há cerca de um mês ter apresentado episódio agudo de febre e mal-estar, tendo procurado atendimento hospitalar após cerca de 5 dias na cidade de Campo Grande (MS), onde foi diagnosticada dengue. Permaneceu alguns dias em repouso, havendo melhora do quadro geral; porém, persistiu com queixa de cefaleia no hemicrânio direito e posteriormente dormência na mão esquerda. Procurou neurologista que chegou a solicitar exames para a coluna e prescreveu associação de medicações contendo carisoprodol, diclofenaco e paracetamol. No dia 9 de março de 2013, viajou para Brasília, quando os familiares passaram a notar certa sonolência e confusão mental. Procuraram atendimento em pronto-socorro na mesma noite, quando o paciente apresentava tremores nas pernas, sendo referido para avaliação psiquiátrica. Após received

May 9, 2013

accepted

August 7, 2015

published online

Novembro 3, 2015
DOI http://dx.doi.org/

10.1055/s-0035-1564827. ISSN 0103-5355.
Copyright $(2016$ by Thieme Publicações License terms Ltda, Rio de Janeiro, Brazil 
cerca de uma semana neste quadro, no dia 17 de março de 2013 os familiares perceberam ao despertar que o paciente havia tido perda urinária e apresentava dificuldade para deambular, com piora do quadro de desorientação temporoespacial. Solicitaram atendimento domiciliar por ambulância que o levou ao serviço de emergência do Hospital das Forças Armadas. Admitido com escala de Glasgow 12 (que após cerca de 20 minutos caiu para 9), hemiparesia esquerda grau 3, com pupilas isométricas e fotorreagentes. A tomografia computadorizada demonstrou a presença de uma coleção subdural com áreas de hiperdensidade e outras com características iso e hipodensas em topografia frontotemporoparietal direita, com desvio de linha média mensurado em $20 \mathrm{~mm}$ e compressão encefálica com apagamento do ventrículo ipsilateral. Os exames laboratoriais apontaram tempo de protrombina de 63,2 segundos, atividade protrombínica de $12 \%$ (valores de referência de 70-100\%) e RNI $=5,68$ (valor de referência até 1,24 ); os demais estudos bioquímicos e hematimétricos apresentavam-se normais. Em função do quadro de rápida deterioração neurológica, o paciente foi levado ao centro cirúrgico, onde se procedeu à administração de cinco ampolas de complexo protrombínico, seguida da realização de duas trepanações (frontal e parietal) com drenagem de hematoma liquefeito com característica hipertensiva. Foi feita lavagem com soro fisiológico até o retorno claro conforme técnica padrão e deixado dreno no espaço subdural, com fechamento da incisão cirúrgica. Não houve sangramento significativo no intraoperatório. 0 paciente foi mantido sob sedação por 24 horas em ambiente de terapia intensiva. Apresentou boa evolução neurológica, com recuperação do déficit motor e da consciência, recebendo alta hospitalar em 21 de março de 2013 . O paciente retornou ao ambulatório em 4 de abril de 2013, apresentando exame neurológico normal.

\section{Discussão}

A ocorrência de hematoma subdural (agudo e crônico) mais comumente ocorre na vigência de traumatismo cranioencefálico, mas há relatos de hematomas espontâneos, geralmente associados à presença de malformações vasculares intracranianas, ${ }^{3}$ hipertensão, ${ }^{4,5}$ coagulopatias, ${ }^{6}$ uso de drogas $^{7}$ ou ruptura arterial cortical. ${ }^{5,8}$ No presente caso foi observada a presença de coagulopatia decorrente do uso de anticoagulante (warfarina) possivelmente potencializado pelo uso concomitante de anti-inflamatório. O conhecimento das interações medicamentosas é relevante, principalmente pela grande frequência de uso de múltiplas medicações, particularmente na população idosa. O mecanismo de ação da warfarina ocorre pela inibição das redutases envolvidas na conversão da vitamina K epóxido em vitamina KH2 (hidroquinona), levando à produção pelo fígado de proteínas descarboxiladas ou parcialmente descarboxiladas com menor percentual de atividade biológica causando a redução na quantidade total dos fatores II, VII, IX e X. ${ }^{9}$

A situação de coagulopatia com necessidade de neurocirurgia de emergência traz sérias implicações inclusive na esfera médico-legal devido à possibilidade de complicações relacionadas a sangramentos no intra e pós-operatório. É importante para o neurocirurgião ter opções para a realização segura da cirurgia nesta situação. Recentemente, o fator protrombínico vem sendo utilizado em procedimentos cirúrgicos, ${ }^{10,11}$ pós-operatório ${ }^{12} \mathrm{e}$ na reversão de anticoagulação no tratamento de acidentes vasculares hemorrágicos. ${ }^{13} \mathrm{O}$ mecanismo de ação deste medicamento se dá através da reposição dos fatores II, VII, IX, X e proteína $\mathrm{C}$, tendo aplicação também no tratamento de hemofilias e outras condições hemorrágicas. ${ }^{14}$

Na condução clínica dos pacientes que desenvolvem este tipo de complicação associada ao uso de warfarina, é importante a suspensão da droga, a administração de vitamina $\mathrm{K}$ e o uso de plasma; porém, em uma situação de emergência neurocirúrgica o uso do complexo protrombínico pode ser mais adequado. Estudos recentes consideram seguro o uso de fator VIIa recombinante em pacientes com hemorragia intracraniana pelo uso de warfarina. ${ }^{15} \mathrm{O}$ grande diferencial destas abordagens seria a rapidez da ação possibilitando a intervenção cirúrgica em um tempo mais curto, ${ }^{16}$ uma vez que a realização precoce da cirurgia é o principal fator prognóstico no tratamento dos hematomas subdurais espontâneos. ${ }^{8,17}$ A interrupção da anticoagulação por um período curto para a realização da cirurgia e pósoperatório é considerada segura do ponto de vista da ocorrência de fenômenos tromboembólicos, mesmo em pacientes portadores de próteses. ${ }^{18} \mathrm{~A}$ interação do neurocirurgião com profissionais de outras especialidades, como intensivistas, clínicos e anestesiologista, é fundamental para uma melhor condução dos pacientes com patologias de tratamento neurocirúrgico.

\section{Referências}

1 Hart RG, Boop BS, Anderson DC. Oral anticoagulants and intracranial hemorrhage. Facts and hypotheses. Stroke 1995;26(8):1471-1477

2 Schulman S, Beyth RJ, Kearon C, Levine MN; American College of Chest Physicians. Hemorrhagic complications of anticoagulant and thrombolytic treatment: American College of Chest Physicians Evidence-Based Clinical Practice Guidelines (8th Edition). Chest 2008;133(6, Suppl)257S-298S

3 Gilad R, Fatterpekar GM, Johnson DM, Patel AB. Migrating subdural hematoma without subarachnoid hemorrhage in the case of a patient with a ruptured aneurysm in the intrasellar anterior communicating artery. AJNR Am J Neuroradiol 2007;28(10):2014-2016

4 Talalla A, McKissock W. Acute "spontaneous" subdural hemorrhage. An unusual form of cerebrovascular accident. Neurology 1971;21(1):19-25

5 Chhiber SS, Singh JP. Acute spontaneous subdural hematoma of arterial origin: a report of four cases and review of literature. Neurol India 2010;58(4):654-658

6 Agrawal D, Mahapatra AK. Spontaneous subdural hematoma in a young adult with hemophilia. Neurol India 2003;51(1):114-115

7 Alves OL, Gomes O. Cocaine-related acute subdural hematoma: an emergent cause of cerebrovascular accident. Acta Neurochir (Wien) 2000;142(7):819-821

8 Gelabert-González M, Fernández-Villa JM, Iglesias-Pais M, González-García J, García-Allut A. Hematoma subdural agudo espontâneo de origen arterial. Neurocirurgía 2004;15:165-170

9 Teles JS, Fukuda EY, Feder D. Warfarin: pharmacological profile and drug interactions with antidepressants. Einstein (Sao Paulo) 2012;10(1):110-115 
10 Bruce D, Nokes TJ. Prothrombin complex concentrate (Beriplex P/ $\mathrm{N}$ ) in severe bleeding: experience in a large tertiary hospital. Crit Care 2008;12(4):R105

11 Schick KS, Fertmann JM, Jauch KW, Hoffmann JN. Prothrombin complex concentrate in surgical patients: retrospective evaluation of vitamin $\mathrm{K}$ antagonist reversal and treatment of severe bleeding. Crit Care 2009;13(6):R191

12 Arnékian V, Camous J, Fattal S, Rézaiguia-Delclaux S, Nottin R, Stéphan F. Use of prothrombin complex concentrate for excessive bleeding after cardiac surgery. Interact Cardiovasc Thorac Surg 2012;15(3):382-389

13 Imberti D, Barillari G, Biasioli C, et al. Emergency reversal of anticoagulation with a three-factor prothrombin complex concentrate in patients with intracranial haemorrhage. Blood Transfus $2011 ; 9(2): 148-155$
14 Chaves DG, Rodrigues CV. Desenvolvimento de inibidores do fator VIII na hemofilia A. Rev Bras Hematol Hemoter. 2009;31(5):384-390

15 H-y CS, Xuemei C, G KR, et al. Thromboembolic risks of recombinant factor VIIa Use in warfarin-associated intracranial hemorrhage: a case-control study. BMC Neurol 2012;12:158

16 Fredriksson K, Norrving B, Strömblad LG. Emergency reversal of anticoagulation after intracerebral hemorrhage. Stroke 1992; 23(7):972-977

17 Akioka N, Fukuda O, Takaba M, Kameda H, Saito T, Endo S. Clinical investigation of acute spontaneous subdural hematoma cases. J Stroke Cerebrovasc Dis 2007;16(3):109-113

18 Chandra D, Gupta A, Grover V, Kumar Gupta V. When should you restart anticoagulation in patients who suffer an intracranial bleed who also have a prosthetic valve? Interact Cardiovasc Thorac Surg 2013;16(4):520-523 